\title{
Mecanismos de transferência de conhecimento interorganizacional: um estudo na maior instituição brasileira de pesquisa agropecuária
}

\author{
DANIELA MARTINS DINIZ ${ }^{1}$ \\ FABRICIO MOLICA DE MENDONÇA ${ }^{1}$ \\ FÁtIMA BAYMA DE OLIVEIRA ${ }^{2}$ \\ ANDERSON DE SOUZA SANT'ANNA ${ }^{3}$
}

\begin{abstract}
${ }^{1}$ Universidade Federal de SÃo JoÃo del ReI (UFSJ) / Departamento de CIÊNCIAS AdMINISTRATIVAS E ContábEIS, SÃo JoÃo dEL REI - MG, BRASIL

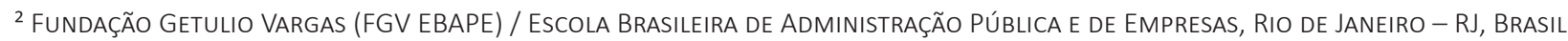

${ }^{3}$ FundaçÃo Getulio Vargas (FGV EAESP) / Escola de AdministraçÃo de EMPRESAS de SÃo PaUlo, SÃo PaUlo - SP, BRASIL
\end{abstract}

\begin{abstract}
Resumo
Baseando-se na perspectiva teórica da transferência de conhecimento interorganizacional, este estudo teve como objetivo analisar o papel dos mecanismos no desempenho da transferência de conhecimento entre uma instituição brasileira de pesquisa pública e suas empresas licenciadas. Em termos metodológicos, foi realizada pesquisa de natureza qualitativa com base no método de estudo de caso aplicado na maior instituição pública de pesquisa agropecuária do Brasil. Os resultados do estudo apontam que, a despeito da diversidade de mecanismos disponíveis, a transferência de conhecimento entre a instituição de pesquisa e as empresas licenciadas é caracterizada pelo uso intensivo de mecanismos de codificação ao invés de mecanismos de personalização, os quais são fundamentais na transferência de conhecimento tácito. Tais achados convergem com estudos na área que sinalizam que o compartilhamento de conhecimento tácito requer o uso de mecanismos de personalização e que, na ausência de mecanismos adequados, a transferência tecnológica tende a enfrentar desafios consideráveis, como a emergência de conflitos relacionais entre as partes e a dificuldade de aplicação da tecnologia pelas empresas receptoras, inibindo o potencial de inovação do processo. Portanto uma implicação relevante é a de que a transferência de conhecimento interorganizacional deve ser concebida como um processo relacional que requer aprendizagem conjunta, sobretudo, quando o tipo de conhecimento envolvido possui natureza complexa.
\end{abstract}

Palavras-chave: Transferência de Conhecimento. Relações Interorganizacionais. Mecanismos de Transferência. Tecnologias Agropecuárias.

Interorganizational knowledge transfer mechanisms: a study in the largest Brazilian institution of agricultural research

\section{Abstract}

Based on the theoretical perspective of interorganizational knowledge transfer, the objective of the study was to analyze the role of mechanisms in the performance of knowledge transfer between a Brazilian public research institution and its licensed companies. In methodological terms, qualitative research was carried out based on the case study method applied in the largest national institution of public agricultural research. The results of the study point out that, despite the diversity of available mechanisms, the knowledge transfer between the Research Institution and the licensed companies is characterized by the intensive use of coding mechanisms rather than customization mechanisms, which are fundamental in transfer of tacit knowledge. These findings converge with studies in the field that indicate that the sharing of tacit knowledge requires the use of personalization mechanisms and that, in the absence of adequate mechanisms, technology transfer tends to face considerable challenges, such as the emergence of relational conflicts in the partnership and the difficulty of applying the technology by the receiving companies, inhibiting the process innovation potential. Therefore, a relevant implication is that the transfer of interorganizational knowledge should be conceived as a relational process that requires joint learning, especially when the type of knowledge involved has a complex nature.

Keywords: Knowledge Transfer. Interorganizational Relationships. Transfer Mechanisms. Agricultural Technologies.

Mecanismos de transferencia de conocimiento interorganizacional: un estudio en la mayor institución brasileña de investigación agropecuaria

\section{Resumen}

Con base en la perspectiva teórica de la transferencia de conocimiento interorganizacional, este estudio tiene como objetivo analizar el papel de los mecanismos en el desempeño de la transferencia de conocimiento entre una institución brasileña de investigación pública y sus empresas licenciadas. En términos metodológicos, se realizó una investigación de naturaleza cualitativa con base en el método de estudio de caso aplicado en la mayor institución brasileña de investigación pública agropecuaria. Los resultados del estudio apuntan que, a pesar de la diversidad de mecanismos disponibles, la transferencia de conocimiento entre la institución de investigación y las empresas licenciadas se caracteriza por el uso intensivo de mecanismos de codificación en lugar de mecanismos de personalización, que son fundamentales en transferencia de conocimiento tácito. Tales hallazgos convergen con diversos estudios en el área que señalan que el compartir de conocimiento tácito requiere el uso de mecanismos de personalización y que, en ausencia de mecanismos adecuados, la transferencia tecnológica tiende a enfrentar desafíos considerables, como la emergencia de conflictos relacionales entre las partes y la dificultad de aplicación de la tecnología por las empresas receptoras, inhibiendo el potencial de innovación del proceso. Por lo tanto una implicación relevante es que la transferencia de conocimiento interorganizacional debe ser concebida como un proceso relacional que requiere aprendizaje conjunto, sobre todo, cuando el tipo de conocimiento involucrado tiene naturaleza compleja.

Palabras clave: Transferencia de conocimiento. Relaciones interorganizacionales. Mecanismos de transferencia. Tecnologías agropecuarias. 


\section{INTRODUÇÃO}

As pesquisas no campo da Teoria das Organizações, a partir da década de 1990, têm privilegiado o tema "conhecimento" em uma série de estudos especialmente ligados a aprendizagem, gestão do conhecimento e da inovação (GRANT, 2002; NONAKA e VON KROGH, 2009). O aumento do interesse em torno dessa temática decorre da constatação de que o conhecimento é um dos recursos críticos das organizações e de que a sua capacidade de gerar novos conhecimentos, gerenciá-los e aplicá-los com êxito é um dos fatores decisivos para a competitividade das empresas no atual contexto de negócios (KOGUT e ZANDER, 1993; ARGOTE e INGRAM, 2000; GRANT, 2002; NONAKA e VON KROGH, 2009; ARGOTE e MIRON-SPEKTOR, 2011; DINIZ, 2011).

Assim, um dos processos que vêm se destacando como um importante modo de aquisição de novos conhecimentos e inovação é a transferência de conhecimento interorganizacional (PÉREZ-NORDTVEDT, KEDIA, DATTA et al., 2008; VAN WIJK, JANSEN e LYLES, 2008; MARTINKENAITE, 2011; BOZEMAN, RIMES e YOUTIE, 2015; BATTISTELLA, DE TONI e PILLON, 2016). Por um lado, a transferência configura-se como uma alternativa de inovação às organizações que não têm condições de desenvolver internamente um programa de Pesquisa e Desenvolvimento (P\&D), em virtude de seu elevado custo, risco e incerteza. Por outro, a transferência também é relevante para as organizações que possuem um programa de P\&D, na medida em que proporciona sentido econômico ao processo de inovação. Ou seja, possibilita à organização difundir a inovação no setor produtivo e obter o retorno do investimento da pesquisa realizada (LOVE e ROPER, 1999).

Com a constatação de seu papel como fonte de inovação, a transferência de conhecimento interorganizacional emerge, na década de 1990, como um tema estratégico na literatura organizacional, passando, então, a ser objeto de inúmeros estudos (BOZEMAN, RIMES e YOUTIE, 2015; BATTISTELLA, DE TONI e PILLON, 2016). Desde então, consolida-se uma agenda de pesquisa cujos interesses principais estão relacionados a: a) atores envolvidos na transferência e suas características; b) fatores que afetam o desempenho da transferência, como distância cultural entre as empresas, motivação das partes, capacidade absortiva da receptora, natureza do relacionamento, etc.; c) fases do processo de transferência; d) canais ou mecanismos pelos quais o conhecimento é transferido; e) desempenho e resultados da transferência (MARTINKENAITE, 2011; BOZEMAN, RIMES e YOUTIE, 2015; BATTISTELLA, DE TONI e PILLON, 2016).

A despeito de sua importância como fonte de inovação e competitividade, diversas pesquisas têm evidenciado as dificuldades enfrentadas pelas organizações na condução de um processo de transferência de conhecimento bem-sucedido. Tal situação pode ser resultante da atuação de fatores e mecanismos que, se não considerados a priori, configuram-se como barreiras no processo e comprometem sua eficácia (LARSSON, BENGTSSON, HENRIKSSON et al., 1998; CUMMINGS e TENG, 2003; MUTHUSAMY e WHITE, 2005; EASTERBY-SMITH, LYLES e TSANG, 2008; PÉREZ-NORDTVEDT, KEDIA, DATTA et al., 2008).

Em relação ao papel dos mecanismos particularmente, os estudos na área apontam que a intensidade e a qualidade da interação e do fluxo de conhecimento entre as partes, na transferência de conhecimento interorganizacional, dependem da seleção adequada de mecanismos, na medida em que eles representam a "ponte" ou os "canais" por meio dos quais o processo se operacionaliza. Na sua ausência, a transferência pode falhar em razão das diferenças culturais, relacionais e de conhecimento entre as empresas fonte e receptora, dificultando o potencial de inovação no processo. Portanto o desempenho do processo de transferência de conhecimento interorganizacional é significativamente afetado pela presença de mecanismos.

Não obstante, estudos na área apontam que a literatura existente ainda é limitada em explicar como empresas fonte e receptora selecionam mecanismos de transferência adequados, bem como a influência da natureza do conhecimento na escolha desses canais (CUMMINGS e TENG 2003; MARTINKENAITE, 2011; BOZEMAN, RIMES e YOUTIE, 2015; BATTISTELLA, DE TONI e PILLON, 2016). Portanto é preciso considerar a necessidade de novos esforços de investigação voltados à compreensão desse fenômeno sob a ótica dos mecanismos.

Nesse contexto, buscou-se, com a presente pesquisa, analisar empiricamente o papel dos mecanismos no desempenho da transferência de conhecimento entre uma instituição pública de agropecuária e suas empresas licenciadas. Para tanto, foi necessário identificar e caracterizar os mecanismos de transferência de conhecimento utilizados pela instituição de pesquisa e analisar como a escolha por determinados tipos de mecanismos influencia o desempenho do processo de transferência de conhecimento.

Em termos metodológicos, a pesquisa é de natureza qualitativa e baseia-se no método de estudo de caso com a realização de doze entrevistas em profundidade com os principais atores envolvidos no processo de transferência tecnológica (YIN, 2005). O objeto empírico selecionado foi um dos centros de pesquisa da maior instituição pública de pesquisa agropecuária do Brasil, cuja missão é desenvolver e transferir novas tecnologias agropecuárias para a sociedade, especificamente cultivares de milho. 
Em termos de relevância teórica, o estudo busca validar empiricamente o importante impacto dos mecanismos no desempenho da transferência de conhecimento interorganizacional. Soma-se a isso o fato de que ainda não há consenso na literatura sobre como selecionar mecanismos efetivos no processo de transferência. Portanto novos esforços de investigação na área podem contribuir, de alguma forma, para minimizar tal lacuna teórica (MARTINKENAITE, 2011; BOZEMAN, RIMES e YOUTIE, 2015).

Complementarmente, o estudo é relevante ao considerar um importante setor da economia brasileira: a agropecuária. Historicamente, o agronegócio desempenha um relevante papel no cenário socioeconômico do país, por ser uma das primeiras atividades desenvolvidas no território brasileiro, representar 6\% do Produto Interno Bruto (PIB) do Brasil e gerar empregabilidade para a população economicamente ativa do país (IBGE, 2016). Ressalta-se, por fim, que o objeto empírico selecionado foi a maior instituição de pesquisa agropecuária do país e a principal geradora de conhecimento científico-tecnológico nesse campo.

Feita a contextualização da pesquisa, o próximo tópico apresenta a fundamentação teórica do estudo.

\section{REFERENCIAL TEÓRICO}

\section{Transferência de conhecimento interorganizacional}

Conceitualmente a transferência de conhecimento interorganizacional tem sido definida de várias maneiras na literatura (BOZEMAN, RIMES e YOUTIE, 2015; BATTISTELLA, DE TONI e PILLON, 2016). Alguns pesquisadores definem transferência como o movimento ou o fluxo de conhecimentos por entre fronteiras organizacionais (EASTERBY-SMITH, LYLES e TSANG, 2008). Outros autores utilizam termos como "acesso" ou "aquisição" de novos conhecimentos por meio de fontes externas (BATTISTELLA, DE TONI e PILLON, 2016). Outra definição associa a transferência a processos de aprendizagem interfirmas (LARSSON, BENGTSSON, HENRIKSSON et al., 1998; POWELL, 1998; MUTHUSAMY e WHITE, 2005). Há, também, pesquisas que relacionam a transferência à difusão de conhecimentos dentro de redes de relações interorganizacionais (POWELL, 1998). Pontos comuns entre essas definições são: a) o envolvimento de, no mínimo, duas organizações (processo que supera as fronteiras organizacionais); b) a natureza relacional e interativa do processo (LARSSON, BENGTSSON, HENRIKSSON et al., 1998; MUTHUSAMY e WHITE, 2005); c) o conhecimento como um, entre os principais recursos, a ser desenvolvido e transferido.

A despeito de sua importância como fonte de inovação e competitividade, diversos estudos apontam que a transferência de conhecimento interorganizacional é um processo marcado por desafios (SZULANSKI, 2000; CUMMINGS e TENG, 2003; EASTERBY-SMITH, LYLES e TSANG, 2008; PÉREZ-NORDTVEDT, KEDIA, DATTA et al., 2008). Primeiro, porque a transferência implica conexão entre duas ou mais organizações, o que requer que barreiras culturais e relacionais sejam superadas para que a troca de conhecimento efetivamente ocorra (LARSSON, BENGTSSON, HENRIKSSON et al., 1998; POWELL, 1998; MUTHUSAMY e WHITE, 2005). Além disso, o desempenho do processo depende da real assimilação e aplicação do conhecimento na empresa receptora, de modo a gerar os resultados esperados. Portanto, mais do que um simples ato de repassar o conhecimento de um contexto para outro, a transferência é um processo de construção social que requer a adaptação e a transformação do conhecimento externo (ARGOTE e MIRON-SPEKTOR, 2011; MARTINKENAITE, 2011; ARGOTE e FAHRENKOPF, 2016).

A transferência de conhecimento interorganizacional é também influenciada pelo tipo de conhecimento a ser transferido. Conhecimentos tácitos e explícitos são transferidos por meio de diferentes mecanismos e em ritmos distintos e, por isso, a sua natureza deve ser considerada no momento da transferência (ARGOTE, MC EVILY e REAGANS, 2003; CUMMINGS e TENG, 2003; JASIMUDDIN, 2007; VAN WIJK, JANSEN e LYLES, 2008; NONAKA, TOYAMA e HIRATA, 2008; NONAKA e VON KROGH, 2009). Finalmente, a seleção de mecanismos de transferência é uma atividade crucial no processo, dado o seu papel de integrar as organizações e de atenuar as barreiras culturais e relacionais existentes entre elas. Os treinamentos, as conversas pessoais e os manuais são alguns exemplos desses mecanismos (BJORKMAN, STAHL e VAARA, 2007; JASIMUDDIN, 2007; KHARABSHEH, 2007; EASTERBY-SMITH, LYLES e TSANG, 2008).

Em relação ao tipo de conhecimento, alguns pesquisadores apontam que tal recurso é permeado por aspectos culturais do contexto de sua origem, podendo parecer pouco familiar e compreensível em outros ambientes (NONAKA, TOYAMA e HIRATA, 2003; 2008; NONAKA e VON KROGH, 2009). Tais características podem dificultar a assimilação e a aplicação do conhecimento externo pela firma receptora e, consequentemente, prejudicar o processo de transferência de conhecimento interorganizacional ao impedir que o conhecimento seja internalizado e explorado (ARGOTE, MC EVILY e REAGANS, 2003; CUMMINGS e TENG, 
2003; EASTERBY-SMITH, LYLES e TSANG, 2008; VAN WIJK, JANSEN e LYLES, 2008; BATTISTELLA, DE TONI e PILLON, 2016). Ainda que se codifique o conhecimento em manuais e procedimentos, a sua plena exploração requer a transferência do seu componente tácito: a experiência detida pelo indivíduo que produziu tal saber (LEMOS, 2008; VAN WIJK, JANSEN e LYLES, 2008).

Dessa perspectiva, um número considerável de pesquisas chama atenção para a influência da natureza do conhecimento no desempenho de sua transferência interorganizacional (SZULANSKI, 2000; ARGOTE, MC EVILY e REAGANS, 2003; CUMMINGS e TENG, 2003; JASIMUDDIN, 2007; EASTERBY-SMITH, LYLES e TSANG, 2008; VAN WIJK, JANSEN e LYLES, 2008; BOZEMAN, RIMES e YOUTIE, 2015; BATTISTELLA, DE TONI e PILLON, 2016). Enquanto alguns conhecimentos podem ser codificados e materializados com mais facilidade, outros estão enraizados nas pessoas, nas rotinas e na cultura da organização, tornando-os um recurso subjetivo e complexo. Assim, alguns estudos apontam que existem dois tipos de conhecimento que se complementam e interagem no contexto das organizações: o tácito e o explícito (EASTERBY-SMITH, LYLES e TSANG, 2008; NONAKA, TOYAMA e HIRATA, 2003; 2008; NONAKA e VON KROGH, 2009).

O conhecimento tácito resulta da combinação de experiências, valores e percepções dos indivíduos, desenvolvidos em um contexto particular, e está relacionado ao 'como fazer' determinada tarefa. A sua natureza pessoal e subjetiva torna-o um conhecimento difícil de ser verbalizado e transferido (LEMOS, 2008; NONAKA, TOYAMA e HIRATA, 2008; NONAKA e VON KROGH, 2009). Portanto o compartilhamento do conhecimento tácito implica interação social, diálogo e troca de experiências, permitindo aos indivíduos transformarem o seu know-how em informações mais fáceis de serem compreendidas por outras pessoas. Requer, então, um processo de construção social, não sendo possível nem substituir, nem replicar exatamente o conhecimento tácito original (NONAKA e TAKEUCHI, 1997; EISENHARDT e SANTOS, 2002; GRANT, 2002). Ao mesmo tempo, na dificuldade de explicitar o conhecimento tácito reside o seu status de ativo protegido e valioso para a organização e, portanto, fonte importante de criação de valor e de competitividade (BRESMAN, BIRKINSHAW e NOBEL, 1999; GRANT, 2002; SZULANSKI, 2000; SIMONIN, 2004).

O conhecimento explícito, por sua vez, é aquele que pode ser articulado na linguagem formal e, pelo fato de não estar associado a experiências pessoais, guarda poucas características de ambiguidade. Portanto tal conhecimento possui uma característica mais objetiva, podendo ser materializado com mais facilidade, por exemplo, em manuais, normas, metodologias e artefatos (KOGUT e ZANDER, 1993; BRESMAN, BIRKINSHAW e NOBEL, 1999; JASIMUDDIN, 2007; NONAKA, TOYAMA e HIRATA, 2008).

Enquanto o conhecimento tácito torna-se coletivo mediante processos de socialização e aprendizagem coletiva (em que o principal ator da transferência é o indivíduo), a transferência de conhecimento explícito pode ocorrer por meio de sistemas tecnológicos e de processos mais formais (BRESMAN, BIRKINSHAW e NOBEL, 1999; GOH, 2002; JASIMUDDIN, 2007; NONAKA, TOYAMA e HIRATA, 2008). A despeito da distinção entre conhecimento tácito e explícito, eles podem ser constantemente transformados dependendo das atividades conduzidas no âmbito da organização. Aliás, a inovação depende dessa conversão contínua do saber tácito em explícito e vice-versa (NONAKA, TOYAMA e HIRATA, 2008; NONAKA e VON KROGH, 2009).

A natureza do conhecimento tem, portanto, implicações importantes na transferência de conhecimento interorganizacional, uma vez que quanto mais tácito o conhecimento, mais difícil tende a ser o seu compartilhamento e vice-versa (ARGOTE, MC EVILY e REAGANS, 2003; LEMOS, 2008; EASTERBY-SMITH, LYLES e TSANG, 2008; VAN WIJK, JANSEN e LYLES, 2008). Além de sua influência direta no desempenho do processo, o tipo de conhecimento é uma variável decisiva na escolha dos mecanismos de transferência, conforme discussão realizada no próximo tópico.

\section{Mecanismos de Transferência}

Mecanismo de transferência pode ser definido como o canal ou o meio pelo qual o conhecimento é desenvolvido e/ou compartilhado entre as organizações (FERDOWS, 2006; JASIMUDDIN, 2007; BOZEMAN, RIMES e YOUTIE, 2015; BATTISTELLA, DE TONI e PILLON, 2016). A sua finalidade é possibilitar algum tipo de conexão (formal ou informal) entre as instituições, permitindo que a transferência de conhecimento seja concretizada. Conversas informais, reuniões, comitês, comunidades de práticas, equipes interfirmas, intercâmbio de pessoal, treinamentos, criação de manuais e de procedimentos são alguns exemplos de mecanismo (JASIMUDDIN, 2007; KHARABSHEH, 2007; BEKKERS e FREITAS, 2008; EASTERBY-SMITH, LYLES e TSANG, 2008; LAWSON, PETERSEN, COUSINS et al., 2009; NECOECHEA-MONDRAGON, PINEDA-DOMÍNGUEZ e SOTO-FLORES, 2013).

O desempenho da transferência de conhecimento interorganizacional depende da presença desses mecanismos na medida em que eles representam, justamente, os canais pelos quais o conhecimento é compartilhado entre as organizações, favorecendo a aproximação das partes no processo e a superação de barreiras culturais. Em alguns casos, possibilitam que 
uma comunidade social seja criada, na qual conhecimentos tácitos e, portanto, valiosos, são transferidos com mais facilidade (BRESMAN, BIRKINSHAW e NOBEL, 1999; ZAHRA e GEORGE, 2002; BJORKMAN, STAHL e VAARA, 2007).

Estudos na área apontam que, dependendo do tipo de conhecimento a ser transferido, alguns mecanismos são mais adequados. Ou seja, quando a transferência envolve predominantemente o conhecimento tácito, os mecanismos de personalização são mais apropriados, ao passo que, quando prevalece troca de conhecimento explícito, mecanismos de codificação são mais eficientes (GOH, 2002; ARGOTE, MC EVILY e REAGANS, 2003; CUMMINGS e TENG, 2003; JASIMUDDIN, 2007). A despeito dessa classificação, os mecanismos podem ser utilizados de modo complementar.

A estratégia de personalização pressupõe que, dada a natureza subjetiva do conhecimento tácito, mecanismos pessoais e informais podem ser mais adequados, pois permitem que os indivíduos interajam pessoalmente, troquem experiências e aprendam a partir da vivência prática (SZULANSKI, 2000; NONAKA, TOYAMA e HIRATA, 2008; LAWSON, PETERSEN, COUSINS et al., 2009; NONAKA e VON KROGH, 2009). Nesse caso, as pessoas são os principais atores da transferência e os mecanismos podem variar entre encontros pessoais e informais, diálogos de grupo, comunidades de práticas, intercâmbio de pessoal, entre outros (BRESMAN, BIRKINSHAW e NOBEL, 1999; GOH, 2002; JANSEN, VAN DEN BOSCH e VOLBERDA, 2005).

As comunidades de prática, por exemplo, constituem uma abordagem apropriada quando a transferência de conhecimento tácito é prioridade. Consiste na reunião de pessoas para tratar de determinados temas organizacionais, reforçando a capacidade dos membros de pensar em conjunto, de trocar experiências e de desenvolver um sentimento de obrigação mútua. Esse mecanismo auxilia na superação das barreiras individuais e sociais fazendo circular o conhecimento dentro e entre as organizações (DISTERER, 2001).

O intercâmbio de pessoas também é um mecanismo apropriado para a transferência de conhecimento tácito, ao permitir a presença de indivíduos da empresa de origem na organização receptora (ou vice-versa), favorecendo o compartilhamento de experiências fundamentais para a aplicação do conhecimento externo (EASTERBY-SMITH, LYLES e TSANG, 2008; LAWSON, PETERSEN, COUSINS et al., 2009; BATTISTELLA, DE TONI e PILLON, 2016). Caso não ocorra o intercâmbio, a firma receptora pode enfrentar dificuldades para compreender e/ou aplicar o conhecimento por, por exemplo, não ter expertise no assunto ou não ter se envolvido nas fases de criação do conhecimento na empresa de origem, dentre outros fatores (EASTERBYSMITH, LYLES e TSANG, 2008).

Portanto, a transferência de pessoal, juntamente com o conhecimento materializado num manual, protótipo ou produto, permite que experiências sejam compartilhadas e que o conhecimento externo seja adaptado para sua utilização em outro contexto (ARGOTE e INGRAM, 2000; EASTERBY-SMITH, LYLES e TSANG, 2008; LAWSON, PETERSEN, COUSINS et al., 2009; BATTISTELLA, DE TONI e PILLON, 2016).

Os mecanismos de personalização consistem, então, em atividades de socialização nas quais os indivíduos encontram, trocam experiências e aprendem a partir da vivência direta e pessoal (ZAHRA e GEORGE, 2002; JANSEN, VAN DEN BOSCH e VOLBERDA, 2005; BJORKMAN, STAHL e VAARA, 2007). Tais práticas contribuem para elevar a conexão social e a confiança entre as partes, possibilitando a criação de um ambiente convergente de ideias e perspectivas. Assim, a socialização é uma das atividades essenciais na transferência de conhecimento tácito.

Enquanto a transferência de conhecimento tácito exige interação social e atividades de socialização, a transferência de formas mais explícitas de conhecimento pode ocorrer por meio de sistemas tecnológicos, processos estruturados e formais, que são alguns dos mecanismos de codificação. Tendo em vista as suas características - saber formal e codificado -, o conhecimento explícito pode ser transformado em artefatos, como regras, procedimentos, protótipos, materiais e manuais. Esses conhecimentos podem ser mais facilmente disseminados dentro e entre organizações (BRESMAN, BIRKINSHAW e NOBEL, 1999; GOH, 2002; JASIMUDDIN, 2007; Kharabsheh, 2007; NONAKA, TOYAMA e HIRATA, 2008; NONAKA e VON KROGH, 2009).

Com base no exposto, o Quadro 1 reúne os principais elementos teóricos ora discutidos. 


\section{Quadro 1}

Tipo de conhecimento e mecanismos da transferência

\begin{tabular}{|c|c|l|}
\hline \multirow{2}{*}{ Item } & Classificação & \multicolumn{1}{c|}{ Descrição } \\
\hline \multirow{3}{*}{ Conhecimento } & Tácito & $\begin{array}{l}\text { - Experiência e know-how } \\
\text { - Natureza complexa e subjetiva } \\
\text { - Mais difícil de ser codificado, compreendido e transferido }\end{array}$ \\
\cline { 2 - 4 } & Explícito & $\begin{array}{l}\text { - Natureza formal e impessoal } \\
\text { - Conhecimento codificado em manuais, procedimentos, fluxogramas e protótipos }\end{array}$ \\
\hline \multirow{2}{*}{ Mecanismo } & \multirow{2}{*}{ Personalização } & $\begin{array}{l}\text { - Mecanismos informais (nos quais as pessoas são os principais atores da transferência) } \\
\text { - Adequado para transferir conhecimento tácito } \\
\text { - Exemplos: intercâmbio de pessoas e comunidades de prática }\end{array}$ \\
\cline { 2 - 4 } & Codificação & $\begin{array}{l}\text { - Mecanismos formais e estruturados } \\
\text { - Adequado para transferir conhecimento explícito } \\
\text { - Exemplo: manuais, procedimentos, fluxogramas, protótipos }\end{array}$ \\
\hline
\end{tabular}

Fonte: Elaborado pelos autores.

Discutidas as bases teóricas da pesquisa, a próxima seção apresenta as escolhas metodológicas.

\section{Método de Pesquisa}

Com o objetivo de analisar o papel dos mecanismos no desempenho da transferência de conhecimento entre uma instituição brasileira de pesquisa pública e suas empresas licenciadas, optou-se por uma pesquisa de natureza qualitativa, com uso do método de estudo de caso (EISENHARDT, 1989; YIN, 2005). Trata-se de uma estratégia adequada de investigação, dado que a transferência de conhecimento interorganizacional é um tema de pesquisa recente e ainda existe pouca convergência em relação a vários aspectos dessa literatura (MARTINKENAITE, 2011; BOZEMAN, RIMES e YOUTIE, 2015; BATTISTELLA, DE TONI e PILLON, 2016). Nesse sentido, o estudo de caso qualitativo possibilitou uma análise em profundidade do fenômeno investigado; um entendimento da transferência sob a perspectiva dos sujeitos diretamente envolvidos no processo; bem como a emergência de padrões, relações e fatores por trás do imediatamente observado.

Um estudo de caso tem como unidades de análise pessoas, situações, programas ou organizações que existem naturalmente ou são delimitadas intelectualmente pelo pesquisador. A seleção do caso em pesquisa qualitativa é uma decisão importante, pois pode impactar diretamente na relevância dos resultados do estudo. Portanto essa escolha não deve ser aleatória, mas intencional, cujo critério deve ser o de se orientar para a riqueza do fenômeno (EISENHARDT, 1989; YIN, 2005).

Com base nessas premissas, o objeto empírico selecionado foi um dos centros de pesquisa da maior instituição pública de pesquisa agropecuária do Brasil, denominada de Instituição de Pesquisa para fins deste estudo. Criada em 1975, esse centro de pesquisa tem como missão viabilizar soluções de pesquisa, desenvolvimento e inovação com ênfase na cultura de milho e sorgo buscando contribuir, de alguma forma, para a sustentabilidade da agricultura brasileira. Trata-se de um caso relevante para investigar o fenômeno em análise por se tratar da maior instituição pública de pesquisa agropecuária do Brasil; por ter como uma das suas principais funções transferir tecnologias (cultivares de milho) para o setor produtivo; e por deter ampla experiência em processos de transferência tecnológica.

Em relação aos instrumentos de coleta de dados, a pesquisa utilizou entrevistas em profundidade, documentos e notas de campo (EISENHARDT, 1989; YIN, 2005). Foram realizadas doze entrevistas, sendo dez com colaboradores da Instituição de Pesquisa e duas com profissionais das empresas licenciadas que adquirem as tecnologias desta instituição (ver Quadro 2). Ressalta-se que, como se trata de pesquisa qualitativa, cujo propósito principal é compreender a percepção dos sujeitos entrevistados em relação à transferência tecnológica, foram considerados os colaboradores que participam diretamente desse processo. Portanto o objetivo não é generalização estatística, mas sim entendimento qualitativo e holístico do processo (YIN, 2005). 


\section{Quadro 2}

Relação de entrevistados

\begin{tabular}{|c|c|}
\hline Entrevistas & Instituição de pesquisa \\
\hline E1 & Área de Negócio Tecnológico \\
\hline E2 & Área de Negócio Tecnológico \\
\hline E3 & Escritório de Transferência de Tecnologia de Sete Lagoas \\
\hline E4 & Área de Negócio Tecnológico \\
\hline E5 & Escritório de Transferência de Tecnologia de Sete Lagoas \\
\hline E6 & Área de Negócio Tecnológico \\
\hline E7 & Escritório de Transferência de Tecnologia do Triângulo Mineiro \\
\hline E8 & Área de Comunicação e Negócio Tecnológico \\
\hline E9 & Área de Comunicação e Negócio Tecnológico \\
\hline E10 & Área de Pesquisa e Desenvolvimento \\
\hline \multicolumn{2}{|r|}{ Empresas Licenciadas } \\
\hline E11 & Ex-diretor de uma empresa licenciada e atual consultor \\
\hline E12 & Diretor de uma empresa licenciada \\
\hline
\end{tabular}

Fonte: Elaborado pelos autores.

Em relação às questões de pesquisa, o roteiro de entrevista contemplou perguntas vinculadas às seguintes categorias: a) processo de transferência de tecnologia entre a Instituição de Pesquisa e as empresas licenciadas (histórico e descrição); b) mecanismos de transferência utilizados e suas características (de personalização ou codificação); c) tipo de conhecimento transferido entre as partes; d) desafios e dificuldades vivenciados no processo.

As doze entrevistas foram gravadas e transcritas para o armazenamento completo dos dados (gerando 350 páginas de transcrição), bem como para facilitar a organização e o tratamento das informações com base em categorias de análise anteriormente evidenciadas. A fase de entrevistas se encerrou no momento em que as linhas de convergência foram reforçadas e quando a realização de novas entrevistas passou a não gerar novas informações adicionais para a compreensão do fenômeno; isto é, quando a saturação teórica ou de sentido foi alcançada (EISENHARDT, 1989; BAUER e GASKELL, 2002). Ressalta-se, ainda, que todas as observações relevantes captadas pelo pesquisador no momento das entrevistas foram registradas em notas de campo e inseridas nas transcrições pertinentes a cada entrevistado. Após a conclusão da etapa de entrevistas, foi feito um painel de apresentação das evidências com os informantes-chave para validar os resultados encontrados, conferindo, assim, maior rigor e confiabilidade à pesquisa (EISENHARDT, 1989).

Em relação aos documentos, foram realizadas consultas a planos, manuais institucionais; boletins de pesquisa \& desenvolvimento; relatórios técnicos e gerenciais; material de divulgação das tecnologias (cultivares de milho) e ao website da instituição, que é muito rico em termos de informações (conforme indicações feitas na seção de análise de dados).

Para o exame dos dados coletados, empregou-se a análise de conteúdo: técnica de análise de materiais textuais, envolvendo atividades de organização, categorização, interpretação e descrição do conteúdo das informações (BARDIN, 1944; BAUER e GASKELL, 2002; FLICK, 2009). O pesquisador busca compreender profundamente o texto, examinar as suas várias dimensões e construir inferências tendo-o por base (BAUER e GASKELL, 2002). Para facilitar a análise de conteúdo, Flick (2009) sugere a elaboração de categorias de análise concebidas com base na literatura e revistas à luz das evidências da pesquisa. Elaboradas as principais categorias teóricas do estudo, o pesquisador pode organizar os dados de acordo com esses construtos, o que Ihe permite ater-se às principais variáveis de interesse da pesquisa.

Com base no exposto, foram estabelecidas as seguintes categorias de análise na pesquisa realizada: processo de transferência tecnológica; natureza do conhecimento; conhecimento tácito e explícito; mecanismos de transferência; mecanismos de 
personalização e codificação. Assim, os dados obtidos foram organizados de acordo com tais categorias, facilitando, desse modo, sua codificação e interpretação (EISENHARDT, 1989). Com isso, foi possível, também, comparar a percepção de diferentes atores acerca de um mesmo fator, bem como realizar a triangulação de fontes de evidências, isto é, confrontar os dados obtidos por meio de diferentes fontes (entrevistas $x$ documentos), conferindo maior confiabilidade e qualidade aos resultados de pesquisa. Essa técnica de articular múltiplas fontes de evidências para a análise de um mesmo fenômeno é um dos tipos de triangulação utilizados nesta pesquisa (JICK, 1979; EISENHARDT, 1989).

Apresentadas as escolhas metodológicas, a próxima seção contempla a análise dos dados empíricos.

\section{ANÁLISE DOS DADOS}

\section{Tipo de conhecimento e mecanismos de transferência da Instituição de Pesquisa}

Como apontando na revisão teórica, um fator relevante na transferência de conhecimento interorganizacional é a natureza do conhecimento envolvido no processo, bem como a sua influência na seleção dos mecanismos de transferência. $O$ foco da pesquisa empírica foi na transferência de uma das tecnologias mais importantes produzidas pela Instituição de Pesquisa investigada (cultivares de milho), que é um dos principais cereais cultivados no mundo e o segundo grão mais produzido no Brasil (atrás apenas da soja). Assim, a cultivar ou semente de milho é o veículo de transferência de tecnologia que é produzido no âmbito da Instituição de Pesquisa e que chega às empresas licenciadas para ser multiplicada e difundida no mercado (documentos 8, 12, 14).

Os dados empíricos apontam que o milho tem sofrido modificações consideráveis ao longo das últimas décadas, tanto do ponto de vista de melhoramento genético (de P\&D), como de desenvolvimento de novas práticas de produção e manejo, ampliando o nível de complexidade da tecnologia. Dentre essas alterações, destacam-se a sua ampla variabilidade genética e os diversos tipos de sementes que podem ser geradas (híbrido simples, duplo, triplo); bem como a diversidade de técnicas de adubação e de produção (documento 8). Só na safra 2014/2015, por exemplo, a Instituição de Pesquisa disponibilizou no mercado 478 sementes de milho (onze a mais do que na safra anterior): 292 transgênicas e 186 convencionais. Além disso, o milho pode ser usado para atender a demandas de diferentes finalidades e regiões geográficas do Brasil, tendo, portanto, que ser adaptável a essas peculiaridades (documentos 4, 12). O relato abaixo ilustra essa complexidade da tecnologia investigada:

E no nosso caso, do milho, é uma coisa biológica. E então a mesma cultivar, dependendo da empresa e da região, ela pode se comportar diferente, pode ser resistente a uma doença aqui e pode não ser lá. Então hoje eu acho que isto é muito mais complexo. Então o milho pode mudar com o tempo, o mesmo produto pode mudar de acordo com as condições. Então é mais complexa esta transferência (E12).

Assim, os achados indicam que a cultivar de milho possui certo nível de complexidade e que o processo de pesquisa e desenvolvimento dessa tecnologia tende a ser complexo. Isso demanda um corpo de pesquisadores altamente qualificado e em diversas especialidades (zootecnistas, biólogos, agrônomos, geneticista, etc.) - documentos 6, 13, 15, 16 -; bem como o envolvimento das empresas licenciadas no processo de pesquisa, de modo a facilitar a internalização, de sua parte, dos conhecimentos tácitos relacionados à aplicação do milho. Caso contrário, como a literatura aponta, a empresa receptora pode enfrentar dificuldades para utilizar a tecnologia adquirida, comprometendo a efetividade da transferência de conhecimento interorganizacional (BRESMAN, BIRKINSHAW e NOBEL, 1999; CUMMINGS E TENG, 2003; VAN WIJK, JANSEN e LYLES, 2008). Do exposto, quanto mais complexo (tácito) o conhecimento, mais difícil tende a ser a sua transferência entre diferentes organizações (NONAKA, TOYAMA e HIRATA, 2008; NONAKA e VON KROGH, 2009), indicando a necessidade de adoção de mecanismos de socialização.

Além de impactar diretamente no desempenho da transferência, o tipo de conhecimento, também, exerce influência na escolha dos mecanismos (JASIMUDDIN, 2007; KHARABSHEH, 2007; BATTISTELLA, DE TONI e PILLON, 2016). No contexto da Instituição de Pesquisa investigada, foram identificados os seguintes mecanismos utilizados no processo de transferência tecnológica (em menor ou maior grau): a) unidade de observação; b) dias de campo; c) reuniões técnicas; d) sistema treino e visita; e) transferência de conhecimento por meio de canais de comunicação de massa (website, boletins técnicos, matérias em rádio, em televisão e jornal) - documentos 3, 14, 17. 
A unidade de observação é um mecanismo usado pela Instituição de Pesquisa para validar as tecnologias e os conhecimentos produzidos pela instituição diante do mercado-alvo. Nessa etapa do processo de P\&D, a Instituição de Pesquisa cede uma nova semente de milho às empresas licenciadas, que testam e avaliam o desempenho da tecnologia em campo próprio, etapa que pode ser acompanhada por pesquisadores da Instituição. Feitos os testes no campo da empresa licenciada, a Instituição de Pesquisa obtém informações importantes para o aprimoramento da tecnologia antes da sua efetiva transferência (documentos 3,8).

Se a unidade de observação é, geralmente, realizada no decorrer do processo de P\&D, o dia de campo é conduzido após a cultivar de milho estar totalmente desenvolvida e disponível para ser transferida ao mercado. 0 dia de campo, portanto, consiste na demonstração, em campo, das características e da forma de manuseio da tecnologia produzida pela Instituição de Pesquisa para técnicos e empresários das empresas licenciadas. Eles podem ocorrer na Instituição de Pesquisa ou no campo das empresas licenciadas, porém envolvendo a experimentação prática das tecnologias na presença dos futuros usuários (documento 3, 9).

Outra forma de transferir os conhecimentos produzidos pela Instituição de Pesquisa é por meio de reuniões técnicas, que são encontros presenciais realizados com o intuito de planejar atividades, identificar demandas e avaliar os resultados alcançados em reunião com pesquisadores da Instituição de Pesquisa e profissionais das empresas licenciadas. Tais encontros são feitos tendo por base demandas pontuais solicitadas pela Instituição de Pesquisa ou pelas empresas licenciadas (documento 9).

O sistema treino e visita, por sua vez, pode ser considerado um dos mecanismos de transferência mais completos, na medida em que busca integrar todos os métodos acima citados. Embora tal mecanismo não possua foco nas empresas licenciadas, mas em todos os atores da cadeia produtiva do milho, ele pode ser desenvolvido com ênfase nas empresas receptoras. 0 "treino" representa as ações de capacitação dos agentes envolvidos, por meio de cursos, dias de campo, seminários, envio de folders, boletins técnicos, dentre outros, enquanto a "visita" consiste no acompanhamento, por pesquisadores da Instituição de Pesquisa, da aplicação das sementes de milho no campo próprio da empresa licenciada (documento 1).

Por fim, uma forma muito utilizada pela Instituição de Pesquisa para transferir conhecimentos é através de canais de comunicação de massa. Com frequência, diretrizes e informações técnicas sobre as tecnologias da Instituição são divulgadas pelo website e por meio de artigos científicos, boletins técnicos, programas de rádio e televisão, jornais e revistas (documentos 3, 10, 11). Uma fonte importante, neste contexto, é a agência de informação da Instituição de Pesquisa, que consiste em uma plataforma web que possibilita a divulgação e o acesso aos conhecimentos gerados na Instituição. As informações são organizadas numa estrutura ramificada, denominada árvore do conhecimento, que contempla uma classificação de acordo com diversos temas, possibilitando ao usuário o acesso a recursos de informação na íntegra (artigos, livros, imagens, áudios, vídeos). Constitui-se, então, num acervo importante de conhecimentos da Instituição de Pesquisa (documentos 5, 7).

Analisando a natureza e as características dos mecanismos acima descritos, observa-se que eles podem variar entre os mais adequados para transferir conhecimento tácito (mecanismos de personalização), e aqueles mais eficientes no compartilhamento do saber explícito (mecanismos de codificação), como pode ser observado na Figura 1. Como será detalhado posteriormente, os dados empíricos revelam que a Instituição de Pesquisa prioriza o uso de canais de comunicação em massa (ou seja, há incidência maior de mecanismos dessa natureza), empregando pouco mecanismos que favorecem a transferência de conhecimento tácito (como o sistema treino e visita e as unidades de observação). 
Figura 1

Mecanismos utilizados pela Instituição de Pesquisa

\begin{tabular}{|c|c|c|c|c|}
\hline \multirow[b]{2}{*}{$\begin{array}{c}\text { Canais de comunicação } \\
\text { em massa }\end{array}$} & & CANISMC & \multirow[b]{2}{*}{$\begin{array}{l}\text { Unidades de } \\
\text { Observação }\end{array}$} & \multirow[b]{2}{*}{$\begin{array}{c}\text { Sistema Treino } \\
\text { e Visita }\end{array}$} \\
\hline & $\begin{array}{l}\text { Reuniões } \\
\text { Técnicas }\end{array}$ & $\begin{array}{l}\text { Dias de } \\
\text { Campo }\end{array}$ & & \\
\hline \multirow[t]{2}{*}{ EXPLÍ́CITO } & & & & \multirow[t]{2}{*}{ TÁCITO } \\
\hline & \multicolumn{2}{|c|}{ TIPO DE CONHECIMENTO } & & \\
\hline
\end{tabular}

Fonte: Elaborada pelos autores.

Considerando a complexidade da tecnologia e do processo de P\&D das cultivares de milho (exposta anteriormente), era de se esperar o uso intenso de mecanismos de personalização complementarmente aos mecanismos de codificação. Ou seja, além da transferência do conhecimento codificado na semente de milho para as empresas licenciadas, seria adequada a realização sistemática de unidades de observação e de treinos/visitas, na medida em que possibilitaria troca de experiências entre as partes, experimentação prática com a nova tecnologia e o compartilhamento de conhecimentos tácitos (SZULANSKI, 2000; NONAKA, TOYAMA e HIRATA, 2008; NONAKA e VON KROGH, 2009), permitindo uma melhor compreensão e aplicação da cultivar de milho pelas empresas licenciadas. Todavia os achados de pesquisa revelam que tais mecanismos (sobretudo, os de personalização) não têm sido utilizados de forma sistemática (E1, E3, E4, E6):

A Instituição de Pesquisa deveria ensinar as empresas, sobretudo as firmas com pouca experiência, todos os aspectos envolvidos na produção do milho como fazer cruzamento de linhagens, a produção de milho simples, milho duplo, triplo, como deve ser montado o campo, as características do campo, quantas linhas de macho, de fêmea, qual a distância entre as plantas, dentre outros. A empresa tem que conhecer a importância de cada uma dessas variáveis na hora da produção. Esse é um papel que a Instituição pode cumprir. Então, eles podem fazer isso e isso não tem sido feito (E2).

Há indícios de que, até a década de 1990, a Instituição de Pesquisa atuava nas empresas licenciadas de forma mais intensiva, mediante visitas técnicas frequentes nos campos das licenciadas, orientando/capacitando os técnicos da empresa e resolvendo problemas conjuntamente. Entretanto, houve mudança no modelo de parceria entre a Instituição de Pesquisa e as empresas, o que, na visão dos entrevistados, reduziu a interação social e a aproximação pessoal entre as partes, bem como diminuiu o uso de mecanismos de personalização (aqueles mais adequados para transferir conhecimento tácito) (E1, E3, E4, E6). Apesar disso, o uso de mecanismos de codificação (que permite a transferência de conhecimento por meio dos canais de comunicação em massa) foi intensificado, desconsiderando, de alguma forma, a complexidade da tecnologia em questão.

Com relação às reuniões técnicas, por exemplo, os entrevistados da Instituição indicam que é um momento para tratar de assuntos meramente formais, não contribuindo para integrar as partes no processo de transferência (E4), opinião que também é compartilhada pelas empresas licenciadas. Na percepção dos entrevistados, existem mecanismos mais eficientes do que as reuniões: "Tem práticas de campo que são muito mais produtivas do que uma reunião, em que todo mundo vem de longe e senta numa sala." (E11). As reuniões que não ocorrem de forma sistemática (somente uma a duas vezes ao ano) não favorecem o vínculo social e a troca de experiências entre profissionais da Instituição de Pesquisa e das empresas licenciadas (E1, E4).

Há reuniões para definir aspectos formais como discussões do plano de trabalho, quantidade de sementes a serem produzidas, dentre outros. Entretanto, reuniões para troca de experiências entre a Instituição de Pesquisa e as licenciadas não ocorrem (E4).

Em termos de treinamentos, os achados revelam que eles são realizados com base em demandas apontadas pelas empresas licenciadas ou por iniciativa da própria Instituição de Pesquisa, quando, por exemplo, do lançamento de uma nova cultivar ou da identificação de um problema frequente no campo das licenciadas (E3, E8, E11). Entretanto, os dados empíricos sinalizam 
que os treinamentos também não têm cumprido o seu papel na transferência de conhecimento. Por um lado, a Instituição de Pesquisa alega que algumas empresas não têm participado das capacitações oferecidas (geralmente as de maior porte) e que as demandas das licenciadas por treinamento não estão chegando ao conhecimento da Instituição (E1, E3, E6, E10). Por outro lado, as empresas licenciadas apontam que os treinamentos são esporádicos e que a metodologia utilizada pela Instituição de Pesquisa não é adequada, pois não envolve atividades de experimentação e aplicação. Desse modo, há indícios de que os treinamentos servem mais para transmitir informações (conhecimentos explícitos) do que efetivamente capacitar (E10, E11), conforme tangenciam os relatos:

Tem uma série de informações que precisamos saber, o negócio é complexo. É uma confusão que demanda experimentação, comparação. E isso tem que ser sistematizado para passar pros licenciados. Mas é descontínuo (E10).

Os treinamentos não ocorrem de forma permanente, mas sim uma ou duas vezes ao ano (E10).

Com relação às unidades de observação, os dados empíricos indicam que é baixo o número de empresas licenciadas que têm conduzido esse experimento em campos próprios e, quando realizam, não fornecem feedback do desempenho dos materiais para a Instituição de Pesquisa. Segundo relato dos entrevistados, tais informações viram "segredo industrial" para as empresas, que não querem ver esses dados disseminados no mercado e em firmas concorrentes (E1, E3, E4, E6, E12):

[...] as empresas que se interessam em fazer a unidade de observação, as melhores, elas costumam segurar esta informação. Já teve casos que visitamos empresas muito boas, com uma unidade de observação excelente e que os dados não vieram para cá. E têm outro extremo também, empresas que não fazem a unidade de observação (E12).

Além disso, a ideia é que, com a realização de unidades de observação, as partes poderiam trocar informações sobre o desempenho das tecnologias, os problemas de campo, dentre outros assuntos, de modo a retornar insights importantes para o processo de $P \& D$, o que não tem acontecido na prática (E2, E3, E5).

Os dados apontam, ainda, que a frequência de realização de determinados mecanismos (sobretudo os de personalização) é considerada aquém da necessária. Em outros termos, treinamentos esporádicos, eventos anuais ou iniciativas isoladas não são suficientes para promover a aproximação entre a Instituição de Pesquisa e as empresas licenciadas, nem o compartilhamento de conhecimentos entre as partes, sobretudo o tácito (E3, E10). Portanto os dados sinalizam que a metodologia de capacitações, visitas e dias de campo precisa ser sistemática, diferentemente do que ocorre na prática, como evidencia este relato: "Depois dos eventos, não há nenhum tipo de reforço por parte da Instituição de Pesquisa. Não tem um acompanhamento para saber se a empresa está fazendo bem feito" (E3).

Ressalta-se, também, que as empresas licenciadas valorizam mais o uso de mecanismos de personalização (como os dias de campo e as visitas técnicas pelos pesquisadores da Instituição de Pesquisa em seus campos) do que mecanismos formais e impessoais (como relatórios técnicos e manuais, mais próximo do que a literatura aponta como mecanismos de codificação), ou seja, “[...] não é uma página que vai dizer como se produz de forma eficiente. É necessária a participação deles nas nossas atividades e isso nós não temos hoje" (E1).

Assim, na perspectiva das empresas, faltam apoio técnico, acompanhamento pessoal e informações críticas sobre as tecnologias lançadas pela Instituição de Pesquisa, conforme explicita o seguinte trecho:

Eu gostaria muito de receber do meu parceiro (da Instituição de Pesquisa) um apoio mais assíduo. Falta informação. Quando a gente recebe uma cultivar de milho nova para produzir, faltam informações básicas [...] Para trabalhar com o produto eu tenho que ter acesso a todas essas informações. Então, o pacote tecnológico da Instituição de Pesquisa ainda tem fraturas, tem falhas que precisavam ser mais bem trabalhadas. Mas aí, quando você chega na Instituição de Pesquisa e fala "precisamos de alguém que faça isso", e aí começa o 'empurra, empurra'. Vai pra pesquisa, vai pra outra área, etc. (E11).

Com base no exposto, observa-se, por um lado, que as empresas não têm utilizado os mecanismos fornecidos pela Instituição de Pesquisa e, por outro, há uma percepção, por parte das empresas licenciadas, de que os mecanismos empregados pela Instituição não são adequados (e nem a frequência com que são realizados). Consequentemente, os mecanismos que poderiam contribuir para uma maior efetividade na transferência de conhecimento (ao integrar as partes no processo, minimizar as 
diferenças culturais entre as organizações e permitir o compartilhamento de conhecimentos tácitos) não têm cumprido o seu papel no processo de transferência investigado nesta pesquisa (JANSEN, VAN DEN BOSCH e VOLBERDA, 2005; JASIMUDDIN, 2007; KHARABSHEH, 2007; BATTISTELLA, DE TONI e PILLON, 2016).

O Quadro 3 reúne aspectos que se configuram como desafios na transferência de conhecimento entre a Instituição de Pesquisa e as empresas licenciadas, no que tange ao tipo de conhecimento e aos mecanismos de transferência:

\section{Quadro 3}

Achados relacionados ao Tipo de Conhecimento e Mecanismos de Transferência

\begin{tabular}{|c|l|}
\hline Variável & \multicolumn{1}{c|}{ Descrição dos achados } \\
\hline $\begin{array}{c}\text { Tipo de } \\
\text { conhecimento }\end{array}$ & $\begin{array}{l}\text { - Complexidade da tecnologia investigada (da cultivar milho) e do seu processo de P\&D. } \\
\text { - O milho é um conhecimento complexo, pois embora a semente seja o principal veículo de transferência } \\
\text { de tecnologia, há conhecimentos tácitos utilizados no processo de P\&D que são fundamentais para a } \\
\text { aplicação posterior da tecnologia pelas empresas licenciadas. }\end{array}$ \\
\hline \multirow{2}{*}{ Mecanismos } & $\begin{array}{l}\text { - Utilização mais intensa de mecanismos de codificação (transferência de conhecimento por meio de } \\
\text { relatórios técnicos, manuais e canais de comunicação em massa). } \\
\text { - Baixa utilização de mecanismos de personalização. } \\
\text { - As empresas licenciadas não têm participado dos mecanismos fornecidos pela Instituição de Pesquisa. }\end{array}$ \\
\hline
\end{tabular}

Fonte: Elaborado pelos autores.

\section{CONSIDERAÇÕES FINAIS}

Baseando-se na perspectiva teórica da transferência de conhecimento interorganizacional, esta pesquisa analisou o papel dos mecanismos no desempenho da transferência de conhecimento entre uma instituição pública de pesquisa agropecuária e suas empresas licenciadas. Os resultados do estudo apontam que, a despeito da diversidade de mecanismos disponíveis (que variam desde reuniões, cursos, visitas, dias de campo, unidades de observação), o processo de transferência entre a Instituição de Pesquisa e as empresas é caracterizado pelo uso mais intensivo de mecanismos de codificação (em lugar dos métodos de personalização, os quais são fundamentais na transferência de conhecimento tácito).

Assim, os achados do estudo revelam que é baixa a frequência com que são realizadas atividades de socialização e experimentação prática envolvendo a Instituição de Pesquisa e as empresas licenciadas, desconsiderando, de alguma forma, que a transferência de conhecimento interorganizacional vai além de uma simples ação de repassar o conhecimento de um contexto para outro, como a expressão "transferência de conhecimento" semanticamente sugere.

Como contraponto a esse entendimento, os resultados da pesquisa evidenciam a questão da dificuldade de "mobilizar" o conhecimento entre diferentes contextos organizacionais na ausência de mecanismos de transferência adequados. Portanto a transferência de conhecimento interorganizacional deve ser concebida como um processo relacional que requer aprendizagem conjunta, sobretudo, quando o tipo de conhecimento envolvido possui natureza complexa, como é o caso das cultivares de milho. Desse modo, ainda que a Instituição de Pesquisa codifique esses conhecimentos em manuais, publicações e fichas técnicas, tais ações não parecem suficientes para permitir uma efetiva exploração da tecnologia no campo das empresas licenciadas.

Do exposto, há evidências de lacunas em relação ao tipo de mecanismo empregado, bem como em relação à frequência de sua utilização, gerando gargalos no processo de transferência de conhecimento interorganizacional, dentre eles: baixa integração social entre as instituições fonte e receptora, dificultando o diálogo e o relacionamento entre as partes; emergência de conflitos relacionais em razão das diferenças culturais entre as instituições; dificuldades de aplicação da tecnologia pelas empresas licenciadas, inibindo o potencial de inovação do processo. Tais dificuldades poderiam ser atenuadas com a adoção de mecanismos de personalização ao possibilitar ampla interação pessoal entre as partes, compartilhamento de experiências e aprendizagem conjunta, permitindo a superação de barreiras culturais e relacionais entre as partes. Portanto, os mecanismos de personalização são variáveis essenciais quando se pensa em processos de transferência de conhecimento bem-sucedidos, sobretudo quando o conhecimento objeto da transferência possui natureza complexa. 
Desse modo, os resultados apresentados convergem com alguns estudos na área de "transferência de conhecimento" que sinalizam que o compartilhamento de conhecimento tácito requer o uso de mecanismos de personalização e que, na ausência de mecanismos adequados, a transferência tecnológica tende a enfrentar desafios consideráveis.

Este estudo, contribui, de alguma forma, em termos teóricos, para o avanço no entendimento da influência dos mecanismos no desempenho da transferência de conhecimento interorganizacional, indicando, ainda, que a escolha inadequada pelo tipo de mecanismo tem implicações negativas para o êxito do processo. Do ponto de vista empírico, a compreensão do processo de transferência e de algumas variáveis que o afetam pode contribuir com subsídios para que a Instituição de Pesquisa e as empresas licenciadas aprimorem o compartilhamento de conhecimento entre as partes, uma vez que há evidências de dificuldades no processo. Especificamente, em termos de mecanismo, os resultados do estudo apontam que pode ser adequado o desenvolvimento de atividades e capacitações com foco nas empresas licenciadas, pois, embora a instituição atenda a diversos públicos, as empresas agropecuárias que adquirem as suas tecnologias são agentes centrais na disseminação das suas inovações pela cadeia agropecuária, além de proporcionarem sentido financeiro aos recursos investidos em P\&D. Sugere-se, ainda, que a Instituição de Pesquisa estimule a realização contínua de mecanismos de personalização que permitam a troca de experiências e o compartilhamento de conhecimentos tácitos entre fonte e recepção, em decorrência da natureza complexa da tecnologia compartilhada.

Nessa perspectiva, a realização sistemática de mecanismos de personalização pode contribuir para o desempenho da transferência ao atenuar diversos problemas críticos observados no caso investigado, isto é, ao ampliar a integração social entre a fonte e as receptoras, ao minimizar a percepção de distância cultural entre as partes e ao permitir o compartilhamento dos conhecimentos tácitos relacionados à tecnologia, possibilitando uma exploração mais efetiva da tecnologia pelas empresas licenciadas.

\section{AGRADECIMENTOS}

Coordenação de Aperfeiçoamento de Pessoal de Nível Superior - Brasil (CAPES) pelo apoio financeiro.

Fundação de Amparo à Pesquisa do Estado de Minas Gerais (FAPEMIG) pelo apoio financeiro.

Conselho Nacional de Desenvolvimento Científico e Tecnológico (CNPq) pelo apoio para a realização da pesquisa. 


\section{REFERÊNCIAS}

ARGOTE, L.; FAHRENKOPF, E. Knowledge transfer in organizations: the roles of members, tasks, tools, and networks. Organizational Behavior and Human Decision Processes, v. 136, p. 146-159, 2016.

ARGOTE, L.; INGRAM, P. Knowledge transfer: a basis for competitive advantage of firms. Organizational Behavior and Human Decision Processes, v. 82, n. 1, p. 150-169, 2000.

ARGOTE, L.; MC EVILY, B.; REAGANS, R. Managing knowledge in organizations: an integrative framework and review of emerging themes. Management Science, v. 49, n. 4, p. 571-582, 2003.

ARGOTE, L.; MIRON-SPEKTOR, E. Organizational Learning: from Experience to Knowledge. Organization Science, v. 22, n. 5, p. 11231137, 2011.

BARDIN, L. Análise de conteúdo. Lisboa: Edições Setenta, 1994.

BATTISTELLA, C.; DE TONI, A.F.; PILLON, R. J. Inter-organisational technology/knowledge transfer: a framework from critical literature review. The Journal of Technology Transfer, v. 41, n. 5, p. 1195 1234, 2016.

BAUER, M. W.; GASKELL, G. Pesquisa Qualitativa com Texto, Imagem e Som: um manual prático. 2. ed. Petrópolis: Vozes, 2002, 516 p.

BEKKERS, R.; FREITAS, I. M. B. Analysing knowledge transfer channels between universities and industry: To what degree do sectors also matter? Research Policy, v. 37, p. 1837-1853, 2008.

BJORKMAN, I.; STAHL, G. K.; VAARA, E. Cultural differences and capability transfer in cross-border acquisitions: the mediating roles of capability complimentarily, absorptive capacity and social integration. Journal of International Business Studies, v. 38, n. 4, p. 658-672, 2007.

BOZEMAN, B.; RIMES, H.; YOUTIE, J. The evolving state-of-the-art in technology transfer research: revisiting the contingent effectiveness model. Research Policy, v. 44, n. 1, p. 34-49, 2015.

BRESMAN, H.; BIRKINSHAW, J.; NOBEL, R. Knowledge transfer in international acquisitions. Journal of international Business Studies, v. 30, n. 3, p. 439-462, 1999.

CUMMINGS, J. L.; TENG; B. S. Transferring R\&D Knowledge: the key factors affecting Knowledge transfer success. Journal of Engineering and Technology Management, v. 20, p. 39-68, 2003.

DINIZ, D. M. O papel dos fatores antecedentes e dos mecanismos no processo de transferência de conhecimento interfirmas: um estudo de caso na Embrapa milho e sorgo. 2011. Dissertação (Mestrado em Administração) - Pontifícia Universidade Católica de Minas Gerais, Belo Horizonte, 2011.

DISTERER, G. Individual and social barriers to knowledge transfer. In: INTERNATIONAL CONFERENCE ON SYSTEM SCIENCES, 34., 2001, Hawaii Proceedings... 2001.

EASTERBY-SMITH, M.; LYLES, M. A.; TSANG, E. W. K. Interorganizational knowledge transfer: current themes and future prospects. Journal of Management Studies, v. 45, n. 4, p. 677-690, jun. 2008.

EISENHARDT, K. M. Building Theories from Case Study Research. Academy of Management Review, n. 4, v. 14, 1989.
FERDOWS, K. Transfer of changing production know-how. Production and Operations Management, v. 15, n. 2, p. 1-9, 2006.

FLICK, U. Introdução à pesquisa qualitativa. Porto Alegre: Artmed, 2009. $405 \mathrm{p}$.

$\mathrm{GOH}, \mathrm{S}$. C. Managing effective knowledge transfer: an integrative framework and some practice implications. Journal of Knowledge Management, v. 6, n. 1, p. 22-30, 2002.

GRANT, R. M. The knowledge-based view of the firm. In: CHOO, C. W; BONTIS, N. (Eds.). The strategic management of intellectual capital and organizational knowledge. Oxford: Oxford University Press, 2002. p. 133-148.

INSTITUTO BRASILEIRO DE GEOGRAFIA E ESTATÍSTICA - IBGE. Tabelas 2016-Sistema de contas nacionais: Brasil. 2016. Disponível em: $<$ https:// www.ibge.gov.br/estatisticas-novoportal/economicas/servicos/9052sistema-de-contas-nacionais-brasil.html $?=\& \mathrm{t}=$ resultados $>$. Acesso em: 18 dez. 2018.

JANSEN, J. J. P.; VAN DEN BOSCH, F. A. J.; VOLBERDA, H. W. Managing potential and realized absorptive capacity: how do organizational antecedents matter? Academy of Management Journal, v. 48, n. 6, p. 999-1015, 2005.

JASIMUDDIN, Sajjad M. Exploring knowledge transfer mechanisms: the case of a UK-based group within a high-tech global corporation. International Journal of Information Management, v. 27, p. 294300, 2007.

JICK, T. D. Mixing qualitative and quantitative methods: triangulation in action. Administrative Science Quarterly, v. 24, 1979.

KHARABSHEH, R. A. A. Model of Antecedents of Knowledge Sharing. The eletronic Journal of Knowledge Managemnet, v. 5, p. 419-426, 2007.

KOGUT, B., ZANDER, U. Knowledge of the firm and the Evolutionary Theory of the multinational corporation. Journal of International Business Studies, v. 24, p. 625-645, 1993.

LARSSON, R. et al. The interorganizational learning dilemma: collective knowledge development in strategic alliances. Organization Science, v. 9 , n. 3, p. 285-305, 1998

LAWSON, B. et al. Knowledge sharing in interorganizational product development teams: the effect of formal and informal socialization mechanisms. Journal Product Innovation Management, v. 26, n. 2, p. 156-172, 2009.

LEMOS, B. N. Fatores relevantes de sucesso à transferência do conhecimento tácito: evidências empírico-exploratórias em uma empresa petrolífera brasileira. 128 p. 2008. Dissertação (Mestrado em Gestão) - Escola Brasileira de Administração Pública e de Empresas, Fundação Getulio Vargas, Rio de Janeiro, 2008.

LOVE, J. H.; ROPER, S. The determinants of innovation: R\&D, technology transfer and networking effects. Review of Industrial Organization, v. 15, p. 43-64, 1999.

MARTINKENAITE, I. Antecedents and consequences of interorganizational knowledge transfer: emerging themes and openings for further research. Baltic Journal of Management, v. 6, n. 1, p. 53-70, 2011. 
Mecanismos de transferência de conhecimento interorganizacional: um estudo na maior instituição brasileira de pesquisa agropecuária
Daniela Martins Diniz

Fabricio Molica de Mendonça

Fátima Bayma de Oliveira | Anderson de Souza Sant'Anna
MUTHUSAMY, S. K.; WHITE, M. A. Learning and knowledge transfer in strategic alliances: a social exchange view. Organization Studies, v. 26, n. 3, p. 415-441, 2005.

NECOECHEA-MONDRAGON, H. et al. A conceptual model of technology transfer for public universities in Mexico. Journal of Technology Management \& Innovation, Santiago, v. 8, n. 4, p. 24-35, 2013.

NONAKA, I.; TAKEUCHI, H. Criação de conhecimento na empresa: como as empresas japonesas geram a dinâmica da inovação. Rio de Janeiro: Campus, 1997. 358p.

NONAKA, I.; TOYAMA, R.; HIRATA, T. Teoria e casos de empresas baseadas no conhecimento: managing flow. Porto Alegre: Bookman, 2003. 303 p.

NONAKA, I.; TOYAMA, R.; HIRATA, T. Managing Flow: a process theory of the knowledge-based firm. New York: Palgrave Macmillan, 2008.

NONAKA, I.; VON KROGH, G. Tacit knowledge and knowledge conversion: controversy and advancement in organizational knowledge Creation Theory. Organization Science, v. 20, n. 3, p. 635-652, 2009.
PÉREZ-NORDTVEDT, L. et al. Effectiveness and Efficiency of CrossBorder Knowledge Transfer: An Empirical Examination. Journal of Management Studies, v. 45, n. 4, p. 714-744, June 2008.

POWELL, W. W. Learning from collaboration: knowledge and networks in the biotechnology and pharmaceutical industries. California Management Review, v. 40, n. 3, p. 228-240, 1998.

SIMONIN, B. An empirical investigation of the process of knowledge transfer in international strategic alliances. Journal of International Business Studies, v. 35, n. 5, p. 407-427, 2004.

SZULANSKI, G. The process of knowledge transfer: A diachronic analysis of stickiness. Organizational Behavior and Human Decision Processes, v. 82, p. 9-27, 2000.

VAN WIJK, R.; JANSEN, J. J. P.; LYLES, M. A. Inter and intra-organizational knowledge transfer: a meta-analytic review and assessment of its antecedents and consequences. Journal of Management Studies, v. 45, n. 4, p. 830-853, June 2008.

YIN, R. K. Estudo de caso: planejamento e métodos. 3. ed. Porto Alegre: Bookman, 2005.

ZAHRA, S. A.; GEORGE, G. Absorptive Capacity: a review, reconceptualization and extension. Academy of Management Review, v. 27, p. 185-203, 2002.

Daniela Martins Diniz

ORCID: https://orcid.org/0000-0001-8535-8703

Professora Adjunta da Universidade Federal de São João Del Rei (UFSJ) vinculada ao Departamento de Ciências Administrativas e Contábeis (DECAC); Professora do Mestrado Profissional em Propriedade Intelectual e Transferência de Tecnologia para a Inovação do campo focal da Universidade Federal de São João Del Rei (UFSJ); Doutora em Administração pela Universidade Federal de Minas Gerais (UFMG); Mestre em Administração pela Pontifícia Universidade Católica de Minas Gerais (PUC Minas), São João del Rei - MG, Brasil. E-mail: danidiniz@ufsj.edu.br

Fabricio Molica de Mendonça

ORCID: http://orcid.org/0000-0002-3175-1064

Bacharel em Administração pela Universidade Federal de Viçosa (UFV); Mestre pela Universidade Federal de Viçosa (UFV); Doutor em Engenharia de Produção pela Universidade Federal do Rio de Janeiro (UFRJ); Colaborador e pesquisador da Universidade Federal do Rio de Janeiro (UFRJ); Professor do programa de Mestrado Acadêmico em Administração do Centro Federal de Educação Tecnológica de Minas Gerais (CEFET-MG); Professor do programa de Mestrado Profissional em Propriedade Intelectual e Transferência de Tecnologia para a Inovação e professor Associado II da Universidade Federal de São João Del-Rei (UFSJ), São João del Rei - MG, Brasil. E-mail: fabriciomolica@ufsj.edu.br

Fátima Bayma de Oliveira

ORCID: https://orcid.org/0000-0001-5158-9546

Doutora em Educação pela Universidade Federal do Rio de Janeiro (UFRJ); Mestre em Administração Pública pela University of Connecticut (EUA); Bacharel em Administração Pública pela Escola Brasileira de Administração Pública e de Empresas da Fundação Getulio Vargas (FGV EBAPE); Professora Titular da Fundação Getulio Vargas (FGV EBAPE) nos cursos de Mestrado e Doutorado em Administração, Rio de Janeiro - RJ, Brasil. E-mail: fatima.oliveira@fgv.br

Anderson de Souza Sant'Anna

ORCID: https://orcid.org/0000-0001-6537-6314

Professor Adjunto pela Escola de Administração de Empresas de São Paulo da Fundação Getulio Vargas (FGV EAESP); Pesquisador Produtividade em Pesquisa do Conselho Nacional de Desenvolvimento Científico e Tecnológico (CNPq); Líder do Observatório de Relações Indivíduo-OrganizaçõesSociedade- ORIOS (FDC-CNPq); Membro da Câmara de Assessoramento da Área de Ciências Sociais Aplicadas da Fundação de Amparo à Pesquisa do Estado de Minas Gerais (FAPEMIG); Pós-doutor pelo Programa de Pós-Graduação em Teoria Psicanalítica da Universidade Federal do Rio de Janeiro (UFRJ); Doutor em Administração; Doutor em Arquitetura e Urbanismo; Mestre em Administração; Especialista em Gestão Estratégica; Bacharel em Administração pela Universidade Federal de Minas Gerais (UFMG), São Paulo - SP, Brasil. E-mail: andersonsant@icloud.com 


\section{APÊNDICE}

\section{Apêndice A \\ Relação de documentos consultados na Instituição de Pesquisa}

\begin{tabular}{|c|c|c|}
\hline $\mathrm{N}^{\circ}$ & Título & Tipo de documento \\
\hline 1 & Manual Treino e Visita & Página web da Instituição de Pesquisa \\
\hline 2 & Política de Negócios Tecnológicos & Política Institucional \\
\hline 3 & Transferência de tecnologia na Instituição de Pesquisa & Documento Institucional \\
\hline 4 & $\begin{array}{l}\text { Transferência de tecnologia em práticas promotoras de rendimento de grãos de } \\
\text { milho }\end{array}$ & Documento Institucional \\
\hline 5 & $\begin{array}{l}\text { Agência de informação: uma ferramenta para gestão do conhecimento em } \\
\text { empresas de PD\&l }\end{array}$ & Artigo com foco na Instituição de Pesquisa \\
\hline 6 & Considerações sobre a manutenção de germoplasma de milho no Brasil & Documento Institucional \\
\hline 7 & Promoção da informação sobre tecnologias e produtos orgânicos & Artigo com foco na Instituição de Pesquisa \\
\hline 8 & Pré-melhoramento, melhoramento e pós-melhoramento: estratégias e desafios & Livro institucional \\
\hline 9 & $\begin{array}{l}\text { Transferência de tecnologia para cultivares desenvolvida pela Instituição de } \\
\text { Pesquisa }\end{array}$ & Artigo com foco na Instituição de Pesquisa \\
\hline 10 & Relatório de Gestão & Documento institucional \\
\hline 11 & V Plano Diretor da Instituição de Pesquisa (2008-2011-2023) & Plano institucional \\
\hline 12 & $\begin{array}{l}\text { Contrato de licenciamento para produção e comercialização de semente da } \\
\text { cultivar de milho BRS }\end{array}$ & Documento Institucional \\
\hline 13 & $\begin{array}{l}\text { Transferência de tecnologia em práticas promotoras de rendimento de grãos } \\
\text { de milho }\end{array}$ & Documento Institucional \\
\hline 14 & Diretrizes para a transferência de tecnologia: modelo de incubação de empresas & Documento Institucional \\
\hline 15 & $\begin{array}{l}\text { Sistema de avaliação de impacto social da inovação tecnológica agropecuária } \\
\text { (Ambitec-Social) }\end{array}$ & Boletim de P\&D \\
\hline 16 & Pré-melhoramento, melhoramento e pós-melhoramento: estratégias e desafios & Livro institucional \\
\hline 17 & Manual de Implantação do Treino e Visita (T\&V) & Manual Institucional \\
\hline
\end{tabular}

Fonte: Elaborado pelos autores. 